«Системні технології» 4 (129) 2020 «System technologies»

DOI 10.34185/1562-9945-4-129-2020-04

УДК 004.942: 519.711 .3

В.В. Огоренко, С.В. Клименко, Д.С. Астахов

\title{
КОМПЬЮТЕРНЫЕ ИНФОРМАЦИОННЫЕ ТЕХНОЛОГИИ ОБРАБОТКИ ИЗМЕРЕНИЙ В ЗАДАЧАХ НАБЛЮДЕНИЯ И КОНТРОЛЯ
}

Анотация. Выборки экспериментальных измерений содержат информацию о состоянии автоматизированных объектов и систем. Путем оценки и сравнения их средних значений, выборочных дисперсий, гистограмм решаются задачи наблюдения и контроля. Трудности имеют место, если выборки короткие и статистические закономерности неизвестны. Учитывая современные возможности аналого-цифрового преобразования и компьютерной обработки экспериментальных выборок измерений, предлагается проверять гипотезы о статистической однородности коротких выборок измерений путем определения среднего квадрата разности их дискретных эмпирических функций распределения вероятностей, сформированных по экспериментальным выборкам. Это аналог критерия Андерсона. Предложен также дискретный аналог критерия Смирнова-Крамера-фон Мизеса. Проведены вычислительные эксперименты, подтверждающие гипотезу, о том, что дискретные модели функции распределения вероятности и предложенный дискретный средний квадрат разности по информативности не отличается от критерия Андерсона и критерия Смирнова-Крамера- фон Мизеса, но значительно проще при практическом применении в задачах проверки гипотез о статистической однородности коротких выборок экспериментальных измерений.

Ключевые слова: информационные технологии, аналого-цифровое преобразование, статистическая однородность, дискретные критерии однородности.

Вступ. Измерение - это основной источник информации о состоянии и качестве разрабатываемых и модернизируемых технических объектов и технологических процессов. Измеренные показатели их характеризующие, являются случайными величинами. Несмотря на большое число методов неразрушающего контроля (оптический, акустический, вибрационный, электромагнитный, интерферо-метрический, термометрический, радиационный, психометрический) и многообразие видов объектов и технологических процессов, выборки измерений их параметров описываются двумя обобщенными классами математических

(C) Огоренко В.В., Клименко С.В., Астахов Д.С., 2020 
«Системні технології» 4 (129) 2020 «System technologies»

моделей: 1) класс независимых измерений информативных параметров, интегрально характеризующих контролируемые объекты; 2) второй класс - случайные величины в последовательности измерений линейнопротяженных технических объектов [1].

В задачах наблюдения и контроля технических объектов выборки измерений сравниваются и по ним принимаются решения о их состоянии. Трудности решения таких задач имеют место, если выборки измерений короткие и их статистические закономерности неизвестные [2]. Они содержат информацию о состоянии объектов, об их устойчивости или изменении. Рассмотрим информационные технологии компьютерной обработки измерений коротких выборок путем формирования и сравнения их дискретных моделей функций распределения вероятностей экспериментальных выборок измерений с неизвестными статистическими закономерностями.

Постановка задачи. Сравнение коротких выборок случайных величин используется в задачах наблюдения за состоянием контролируемых технических объектов путем анализа экспериментальных измерений параметров с неизвестными статистическими закономерностями. Для их оценки формируются эмпирические непрерывные функции распределения вероятности [3]

$$
F^{*}(x)=\frac{1}{N} \sum_{i=1}^{N} \operatorname{sgn}\left(x-x^{*}(i)\right),
$$

где $x^{*}(i)$ упорядоченная выборка случайных величин, обладающая свойством $x^{*}(1)<x^{*}(2)<<x^{*}(3) \ldots<x^{*}(n-1)<x^{*}(n)$, сформированная по выборке экспериментальных измерений $x(1) x(2) \ldots x(k) \ldots x(n-1) x(n)$.

Для преобразования выборки $X(k)$ в выборку $X^{*}(i)$ используется следующий алгоритм. Сначала определяются собственные ранги измерений $x(k)=x(i)[2]$

$$
R(x(k))=\sum_{i=1}^{N} \operatorname{sgn}(x(k)-x(j)),
$$

где $\operatorname{sgn}(x-a)$ - это функция единичного скачка, равная 1 , если $x(k) \geq a$, и нулю, если $x<a$. 
«Системні технології» 4 (129) 2020 «System technologies»

Затем формируются упорядоченные выборки измерений $x^{*}(i)$ по формуле

$$
x^{*}(i)=x(k) \sum_{k=1}^{N}(\operatorname{sgn}(i-R(x(k)))-\operatorname{sgn}(i-1-R(x(k)))) .
$$

Модели (1) используются для проверки гипотезы о том, что $F^{*}(x)$ - это оценка неизвестной непрерывной функции распределения вероятности $F(x)$ и для проверки гипотезы о том, что две экспериментальные выборки измерений $x_{1}(k)$ и $x_{2}(k)$ - однородные и их функции распределения равны

$$
F_{1}(x(k))=F_{2}(x(k))=F(x(k)) .
$$

Для проверки гипотезы о функции $F^{*}(x)$ используется критерий Смирнова-Крамара-фон Мизеса [3]

$$
V_{c}=\int_{-\infty}^{\infty}\left(F(x)-F^{*}(x)\right)^{2} W(x) d x, \quad W(x)=\frac{d F(x)}{d x} .
$$

Это математическое ожидание квадрата разности теоретической и экспериментальной непрерывных функций распределения вероятности. Заменив в (3) $F^{*}(x)$ формулой (1) и вычислив интеграл, получим

$$
V_{\mathrm{c}}=\sum_{i=1}^{n}\left(F\left(x^{*}(i)\right)-\frac{2 i-1}{2 n}\right)^{2}+\frac{1}{12 n} .
$$

Критерий $V_{\text {c }}$ - случайная величина, порождаемая упорядоченной выборкой случайных величин $x^{*}(i)$. Ее математическое ожидание и дисперсия равны $M\left[V_{\mathrm{c}}\right]=1 / 6, D\left[V_{\mathrm{c}}\right]=1 / 45$, функция распределения вероятности $F_{\mathrm{c}}(V)$ табулирована. Гипотеза о правильном выборе функции $F(x)$ подтверждается, если экспериментальное значение $V_{\mathrm{c}} \leq V_{0}$, где $V_{0}-$ это пороговое значение.

В таблице 1 приведены фрагменты табулированных зависимостей $P\left(V_{0}\right)$ вероятности того, что если $V_{\text {c }} \leq V_{0}$, то с вероятностью $P_{0}=P\left(V_{0}\right)$ принимается решение о правильном выборе функции $F(x)$ при $n \geq 40$ [3]. 
«Системні технології» 4 (129) 2020 «System technologies»

Таблица 1

Фрагменты табулированных зависимостей $P\left(V_{0}\right)$

\begin{tabular}{|c|c|c|c|c|c|}
\hline$P_{0}$ & 0,95 & 0,975 & 0,99 & 0,995 & 0,999 \\
\hline$V_{0}$ & 0,461 & 0,549 & 0,620 & 0,743 & 0,864 \\
\hline
\end{tabular}

Для коротких выборок, $N<40$, порог сравнения $V_{01}$ вычисляется по формуле

$$
V_{01}=\left(1+\frac{1}{n}\right)\left(V_{0}-\frac{0,4}{n}+\frac{0,6}{n^{2}}\right) .
$$

Для проверки гипотезы о том, что две экспериментальные выборки случайных величин $X_{1}(k)$ и $X_{2}(k)$ однородные и описываются одной и той же функцией распределения вероятности $F(x)$, используется классический метод сравнения разностей их квадратов непрерывных эмпирических функций распределения вероятностей $F_{1}^{*}(x)$ и $F_{2}^{*}(x)$, сформированных по выборкам $X_{1}(k)$ и $X_{2}(k)$ [4]

$$
V_{\mathrm{a}}=\frac{1}{2} \int_{-\infty}^{\infty}\left(F_{1}^{*}(x)-F_{2}^{*}(x)\right)^{2} W_{12}(x) d x,
$$

где $W_{12}(x)$ - эмпирический закон распределения вероятностей

$$
W_{12}^{*}(x)=\frac{1}{2}\left(\frac{d F_{1}^{*}(x)}{d x}-\frac{d F_{2}^{*}(x)}{d x}\right)=\frac{1}{2 n} \sum_{i=1}^{n}\left(\delta\left(x-x_{1}(i)\right)+\delta\left(x-x_{2}(i)\right)\right),
$$

где $\delta(x-a)$ - функция Дирака.

После вычисления интеграла (5), получена формула Андерсона

$$
V_{\mathrm{a}}=\frac{1}{2 n^{2}}\left[\sum_{i=1}^{n}\left(R_{0}\left(x_{1}^{*}(i)-i\right)\right)^{2}+\sum_{j=1}^{n}\left(R_{0}\left(x_{2}^{*}(i)-i\right)\right)^{2}\right]-\frac{4 n^{2}-1}{12 n},
$$

где $R_{0}\left(x_{1}^{*}(i)\right)$ и $R_{0}\left(x_{2}^{*}(i)\right)$ - ранги упорядоченных измерений $x_{1}^{*}(i)$ и $x_{2}^{*}(i)$ в объединенной выборке $X_{0}(j)=X_{1}(j)+X_{2}(j-n), k=1,2, \ldots, 2 n$

$$
R_{0}\left(x_{1}^{*}(i)\right)=\sum_{i=1}^{2 n} \operatorname{sgn}\left(x_{1}^{*}(i)-x_{0}(i)\right), R_{0}\left(x_{2}^{*}(i)\right)=\sum_{i=1}^{2 n} \operatorname{sgn}\left(x_{2}^{*}(i)-x_{0}(i)\right) .
$$

Статистика критерия Андерсона практически не отличается от статистики критерия Смирнова-Крамера-фон Мизиса. Его математическое ожидание равно 
«Системні технології» 4 (129) 2020 «System technologies»

$$
M\left[V_{a}^{*}\right]=\frac{1}{6}\left(1+\frac{1}{2 N}\right) .
$$

При компьютерной обработке измерений непрерывные функции $f(x)$ преобразуются в дискретные $f(\Delta x k)$, где $\Delta x$ - это параметр аналого-дискретного преобразования (АЦП). Если в формуле (1) заменить $x$ на $\Delta x k$, то получим эмпирическую дискретную функцию распределения вероятности неупорядоченных измерений $x(j)=x(k), j=k$

$$
F^{*}(\Delta x k)=\frac{1}{n} \sum_{k=1}^{n} s g n(\Delta x k-x(j))
$$

где $\Delta x$ - определяется по формуле $\Delta x n=x_{\max }-x_{\min }$.

Исследуем информативность их использования при решении задач сравнения экспериментальных выборок измерений с неизвестными статистическими закономерностями.

Исследование статистических закономерностей дискретных функций распределения вероятностей. Если известен закон распределения вероятности $W(x)=W(x(j))$, то математическое ожидание и дисперсия случайной величины можно вычислить, учитывая, что $\operatorname{sgn}(\Delta x k \geq x(j))=1$

$$
M\left[F^{*}(\Delta x k)\right]=\int_{-\infty}^{\infty} \operatorname{sgn}(\Delta x k-x) W(x) d x=\int_{-\infty}^{\Delta x k} W(x) d x=F(\Delta x k) .
$$

Следовательно, $F^{*}(\Delta x k)$ - это несмещенная оценка неизвестной функции распределения вероятности $F(x)$ исследуемой выборки независимых случайных величин $x(1) x(2) \ldots x(k) \ldots x(N-1) x(N)$ в интервале значениЙ от $x_{\min }=x^{*}(1)$ до $x_{\max }=x^{*}(N)$ и $\Delta x n=x_{\max }-x_{\min }$.

Дисперсию $F^{*}(\Delta x k)$ определим по формуле

$$
\begin{gathered}
D\left[F^{*}(\Delta x k)\right]=M\left[\left(F^{*}(\Delta x k)\right)^{2}\right]-M^{2}\left[F^{*}(\Delta x k)\right], \\
M\left[\left(F^{*}(\Delta x k)\right)^{2}\right]=\int_{-\infty}^{\infty}(\operatorname{sgn}(\Delta x k-x))^{2} W(x) d x .
\end{gathered}
$$

После вычисления интеграла получим формулу для дисперсии дискретной функции распределения вероятности

$$
D\left[F^{*}(\Delta x k)\right]=\frac{1}{n} F(\Delta x k)(1-F(\Delta x k)) .
$$


«Системні технології» 4 (129) 2020 «System technologies»

Дискретным аналогом критерия $V_{\text {c }}$ проверки гипотезы о статистических закономерностях экспериментальных выборок может служить среднее значение квадрата разности дискретных функций $F(\Delta x k)$ и ее оценки $F^{*}(\Delta x k)$

$$
V_{m}=\sum_{k=1}^{n}\left(F(\Delta x k)-F^{*}(\Delta x k)\right)^{2} .
$$

Определим математическое ожидание дискретного критерия $V_{m}$

$$
M\left[V_{m}\right]=\sum_{k=1}^{n}\left(F^{2}(\Delta x k)-2 F^{*}(\Delta x k) M\left[F^{*}(\Delta x k)\right]+M\left[\left(F^{*}(\Delta x k)\right)^{2}\right]\right) .
$$

После вычисления математических ожиданий получим формулу

$$
M\left[V_{m}\right]=\frac{1}{n} \sum_{k=1}^{n} F(\Delta x k)(1-F(\Delta x k)) .
$$

При функциональных преобразованиях случайной величины вида $y=f(x)$ соблюдается закон сохранения вероятности

$$
W(y) d y=W(x) d x, W(x)=W(y) \frac{d y}{d x} .
$$

Если $f(x)=F(x)$, то $d y / d x=d F(x) / d x=W(x)$ и $W(y)=1$ и функция распределения вероятности $F(y)=y$ в интервале $0 \leq y \leq 1$. Следовательно, если гипотеза подтверждается, то дискретная функция распределения вероятности запишется в виде $F(\Delta x k)=k / n$. Математическое ожидание $M\left[V_{m}\right]$ определяется по формуле

$$
M\left[V_{m}\right]=\frac{1}{N} \sum_{k=1}^{n} \frac{k}{n}\left(1-\frac{k}{n}\right)=\frac{1}{n}\left(\frac{n(n+1)}{2 n}-\frac{n(n+1)(2 n+1)}{6 n^{2}}\right)=\frac{1}{6}\left(\frac{n}{N}-\frac{1}{n N}\right) .
$$

Математическое ожидание дискретного критерия проверки гипотезы $V_{m}$ при $n>10$ практически не отличается по своей информативности от критерия $V_{\mathrm{c}}$, но значительно проще при вычислении.

\section{Исследование информативности дискретных критериев.} Аналогом критерия Андерсона (5) может быть среднее значение квадрата разности двух дискретных эмпирических функций распределения вероятности выборок $X_{1}(k)$ и $X_{2}(k)$

$$
\overline{V_{m}^{*}}=\frac{1}{2} \sum_{k=1}^{n}\left(F_{1}^{*}(\Delta x k)-F_{2}^{*}(\Delta x k)\right)^{2} .
$$


«Системні технології» 4 (129) 2020 «System technologies»

Если функции $F_{1}(x)$ и $F_{2}(x)$ (с*) независимые и однородные, то $M\left[F_{1}^{*}(\Delta x k)\right]=M\left[F_{2}^{*}(\Delta x k)\right]=F(\Delta x k)$. Формулу (13) можно представить в виде

$$
\overline{V_{m}^{*}}=\frac{1}{2} \sum_{k=1}^{n}\left(\Delta F_{1}^{*}(\Delta x k)-\Delta F_{2}^{*}(\Delta x k)\right)^{2}
$$

Следовательно, математическое ожидание $M\left[\overline{V_{m}}\right]$ равно дисперсии $F^{*}(\Delta x k)$

$$
M\left[\overline{V_{m}^{*}}\right]=D\left[F^{*}(\Delta x k)\right]=\frac{1}{n} \sum_{k=1}^{n} F(\Delta x k)(1-F(\Delta x k))=\frac{1}{6}\left(1-\frac{1}{n^{2}}\right) .
$$

Сравнивая математические ожидания $M\left[V_{\mathrm{a}}\right]$ и $M\left[\overline{V_{m}^{*}}\right]$ (формулы (7) и (14), можно сделать вывод, что информативность критериев $\overline{V_{\text {a }}}$ и $\overline{V_{m}}$ по сравнению с информативностью критерия $\overline{V_{c}}$ описывается неравенством $\overline{V_{m}}<V_{\text {c }}<\overline{V_{\text {a }}}$ и алгоритм вычисления $\overline{V_{m}}$ как разности дискретных функций распределения вероятностей значительно проще, чем алгоритм вычисления $\overline{V_{\text {a }}}$.

\section{Исследование информативности дискретных критериев пу-} тем проведения вычислительных экспериментов. Применение рассмотренных дискретных критериев для исследования однородности экспериментальных измерений с неизвестными статистическими закономерностями, размеры выборок которых больше $(N>40)$, не вызывает сомнений [3]. Информативность коротких выборок теоретически подтверждается только путем сравнения математических ожиданий. Исследуем статистические закономерности дискретных критериев путем проведения вычислительных экспериментов на коротких выборках независимых случайных величин, формируемых компьютерными программами. Теоретически их информативность не зависит от вида статистических закономерностей измерений (законов распределения вероятности).

Проведем вычислительные эксперименты на выборках двух видов - с симметричными и ассиметричными законами.

Из симметричных выберем случайные величины с логистической функцией распределения вероятности, из ассиметричных - случайные величины с функцией распределения Релея 
«Системні технології» 4 (129) 2020 «System technologies»

$$
F_{1}(x)=\left(1+\exp \left(-\frac{(x-a) \pi}{\sqrt{3 D}}\right)\right)^{-1}, \quad F_{2}(x)=1-\exp \left(-\frac{x^{2}}{2 b^{2}}\right),
$$

в интервале значений $x_{\max } \leq x \leq x_{\min }$. Их математические ожидания и дисперсии равны:

$$
\begin{gathered}
M_{1}[x]=a, D_{1}[x]=D, x_{\max }=a+3 \sqrt{D}, x_{\min }=0, \\
M_{2}[x]=b \sqrt{\frac{\pi}{2}}, D_{2}[x]=\frac{4-\pi}{2} b^{2}, b=\frac{x_{\max }{ }^{2}}{2 \ln \left((1-p)^{-1}\right)}=\frac{x_{\max }^{2}}{9} .
\end{gathered}
$$

Если выбрать $a=3, \sqrt{D}=1$, то $x_{\max }=6$ и $b=2$.

Формировались выборки независимых случайных величин размером $\mathrm{n}=8,10,20,30$ и оценивались их гистограммы.

На рис.1. представлены гистограммы дискретного критерия $V_{m}$ проверки гипотезы о том, что экспериментальные измерения - это случайные величины, статистические закономерности которых описываются логистической и релеевской функцией распределения вероятности. В соотвествии с данными таблицы 1 значения $V_{m}$ с вероятностями $P_{0}=0,995$ меньше 0,743. По результатам визуального анализа гистонрамм выборок $\mathrm{n}=8$ они меньше 0,5; при $\mathrm{n}=10$ они меньше 0,46; при $\mathrm{n}=20$ и $\mathrm{n}=30$ меньше 0,4; так что по информативности решения задач проверки гипотез о их статистических закономерностях.

Предложенный дискретный критерий может применяться при проведении вычислительных экпериментов для проверки программ формирования выборок случайных величин с заданным законом распределения вероятностей. 
«Системні технології» 4 (129) 2020 «System technologies»
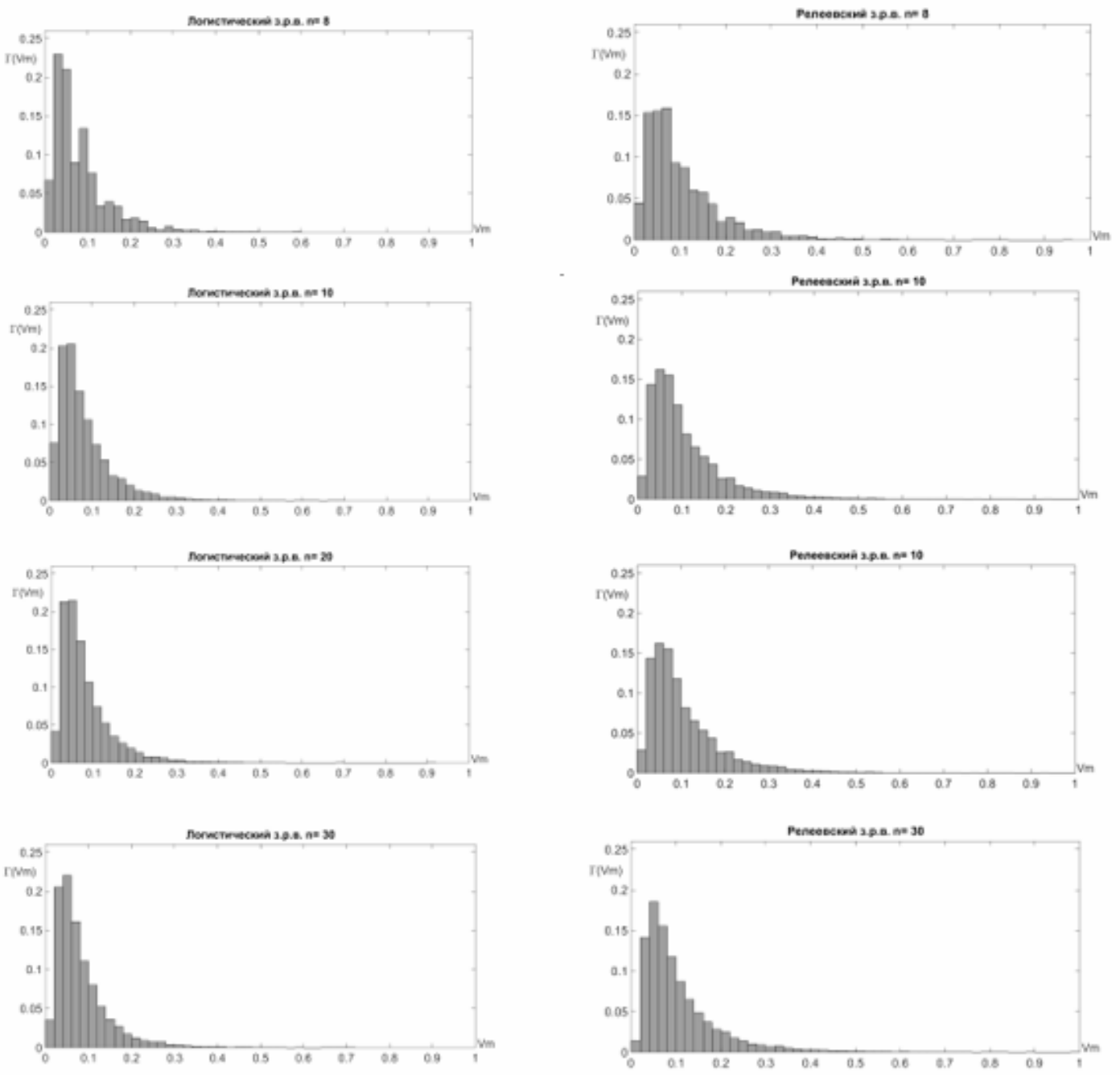

Рисунок 1 - Гистограммы дискретного критерия, аналога критерия Смирнова-Крамера-фон Мизеса

На рис.2 представлены гистограммы дискретного выборочного критерия, аналога критерия Андерсона. По своей информативности они почти не отличаются от информативности одновыборочного критерия n-омега-квадрат. Их можно использовать в задачах наблюдения за техническими объектами путем сравнения двух коротких экспериментальных выборок измерений информативных параметров этих объектов. 
«Системні технології» 4 (129) 2020 «System technologies»
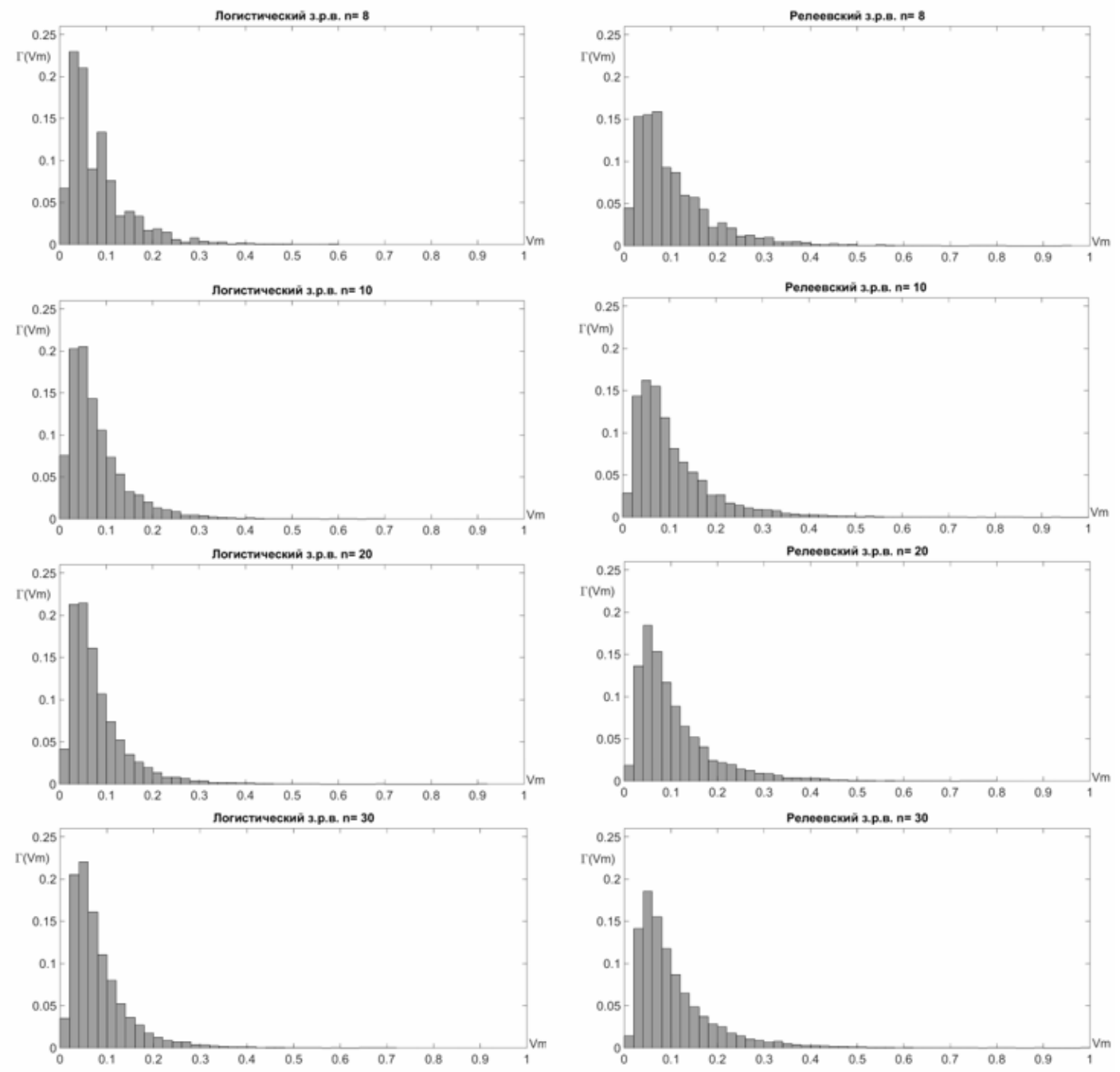

Рисунок 2 - Гистограммы дискретного двухвыборочного критерия, аналога критерия Андерсона

\section{Выводы:}

1. В задачах наблюдения и контроля за состоянием объектов сравниваются короткие выборки измерений их параметров с неизвестными статистическими закономерностями. Классическая математическая статистика предлагает для их решения двухвыборочный критерий Андерсона - эмпирическое математическое ожидание разности двух непрерывных функций распеделения вероятности, формируемых по упорядоченным выборкам экспериметальных измерений. Предложено решение этих задач путем формирования дискретных функций распределения вероятности экспериментальных выборок измерений и оценки среднего квадрата их разности. Это аналог критерия Андерсона. 
«Системні технології» 4 (129) 2020 «System technologies»

2. Для проверки гипотез о статистических закономерностей экспериментальных измерений, их непрерывных функций распредеелния вероятности, вместо критерия Смирнова-Крамора- фон Мизеса применять более постой дискретный критерий - среднее значение квадрата разности теоретической функции распределения вероятности и дискретной функции, сформированной по экспериментальным измерениЯM.

3. Путем проведения вычислительных экспериментов исследованы статистические закономерности дискретных критериев сравнения коротких экспериментальных выборок измерений с симметричным (нормальным, логистическим) и ассиметричным (релеевским) законами распределения вероятностей. Проанализированы гистограммы дискретных критериев оценки их статистической однородности. Статистические закономерности дискретных критериев не отличаются от статистических закономерностей критерия Андерсона и критерия Смирнова - Крамера фон Мизеса, но практическое приминение в задачах проверки гипотез значительно проще, чем их аналогов.

\section{ЛІТЕРАТУРА / ЛИТЕРАТУРА}

1. Технические средства диагностики: Справочник / В.В. Клюев и др.// Под общей редакцией В.В. Клюева. - Машиностроение, 1989. -672с.

2. Гаек Я., Шидак 3. Теория ранговых критериев. - М. Наука, 1971. - 775с.

3. Большев Л.Н. Таблицы математической статистики [текст] / Л.Н. Большев, Н.В. Смирнов. - М.: Наука, 1985. - 416с.

4. Кобзарь А.И. Прикладная математическая статистика. Для инженеров и научных работников. М.: Физматлит, 2006. - 816 с.

\section{REFERENCE}

1. Tehnicheskie sredstva diagnostiki: Spravochnik / V.V. Kljuev i dr.// Pod obshhej redakciej V.V. Kljueva. - Mashinostroenie, 1989. -672s.

2. Gaek Ja., Shidak Z. Teorija rangovyh kriteriev. - M. Nauka, 1971. - 775s.

3. Bol'shev L.N. Tablicy matematicheskoj statistiki [tekst] / L.N. Bol'shev, N.V. Smirnov. - M.: Nauka, 1985. - 416s.

4. Kobzar' A.I. Prikladnaja matematicheskaja statistika. Dlja inzhenerov i nauchnyh rabotnikov. M.: Fizmatlit, 2006. - 816 s. 


\section{«Системні технології» 4 (129) 2020 «System technologies»}

\section{Комп'ютерні інформаційні технології обробки вимірювань \\ в задачах спостереження та контролю}

Вибірки експериментальних вимірювань містять інформацію про стан автоматизованих систем. Шляхом оцінки та порівняння їх середніх значень, вибіркових дисперсій, гістограм вирішуються завдання спостереження за їх станом. Труднощі їх вирішення мають місце, якщо вибірки короткі і статистичні закономірності невідомі. 3 огляду на сучасні можливості аналого-цифрового перетворення та комп'ютерної обробки експериментальних вибірок вимірювань, пропонується перевіряти гіпотези про статистичний однорідності коротких вибірок вимірювань шляхом визначення середнього квадрата різниці їх дискретних емпіричних функцій розподілу ймовірностей, сформованих за експериментальними вибірках. Це двухвибірковий критерій - аналог критерію Андерсона. Запропоновано також одновибірковий дискретний критерій - аналог критерію СмирноваКрамера-фон Мізеса. Проведено обчислювальні експерименти, що підтверджують висновок про те, що дискретні моделі функції розподілу ймовірності та запропоновані дискретні одновибірковий $і$ двухвибірковий критерії по інформативності не відрізняються від критерію Андерсона та критерію Смирнова-Крамера- фон Мізеса, але значно простіше при практичному застосуванні в задачах перевірки гіпотез про статистичні однорідності коротких вибірок експериментальних вимірювань.

\section{Computer information technology for processing measurements in monitoring and control tasks}

Measurement is the main source of information about the condition and quality of developed and modernized technical objects and technological processes. The measured indicators characterizing them are random variables. In the tasks of observation and control of technical objects, measurement samples are compared, and decisions are made on them about their condition. Difficulties in solving such problems occur if the measurement samples are short and their statistical laws are unknown. They contain information about the state of objects, about their stability or change.

Samples of experimental measurements contain information about the state of automated systems. By evaluating and comparing their average values, sample variances, histograms, the tasks of observing their state are solved. Difficulties occur if the samples are short and statistical patterns are unknown.

Classical mathematical statistics offers for their solution the two-sample Anderson criterion - the empirical expectation of the difference of two continuous probability distribution functions formed from ordered samples of experimental measurements. A solution to these problems is proposed by forming discrete probability distribution functions of experimental measurement samples and estimating the mean square of their difference. This is an analogue of the Anderson criterion.

To test hypotheses about the statistical laws of experimental measurements, their continuous functions of probability distribution, instead of the Smirnov-Kramora-von Mises criterion, apply a more consistent discrete criterion - the average value of the square of the difference of the theoretical probability distribution function and the discrete function generated from the experimental measurements.

By means of computational experiments, the statistical laws of discrete criteria for comparing short experimental measurement samples with symmetric (normal, logistic) and asymmetric (Rayleigh) probability distribution laws have been investigated. The histograms of discrete criteria for assessing their statistical homogeneity are analyzed. The statistical laws of 
«Системні технології» 4 (129) 2020 «System technologies»

the discrete criteria do not differ from the statistical laws of the Anderson criterion and the Smirnov-Cramer-von Mises criterion, but practical application in the tasks of testing hypotheses is much simpler than their analogues.

Огоренко Виктория Викторовна - д.мед.н., доцент, Профессор кафедры психиатрии, наркологии и медицинской психологии, Государственное учреждение «Днепровская медицинская академия министерства здравоохранения Украины».

Клименко Светлана Владимировна - к.т.н., доцент, доцент кафедры радиоэлектронной автоматики, Днепровского национального университета имени Олеся Гончара, Украина.

Астахов Дмитрий Сергеевич - старший преподаватель кафедры радиоэлектронной автоматики, физико-технического факультета, Днепровского национального университета имени Олеся Гончара, Украина.

Огоренко Вікторія Вікторівна - д.мед.н., доцент, професор кафедри психіатрії, наркології та медичної психології, Державний заклад «Дніпровська медична академія міністерства охорони здоров'я України».

Клименко Світлана Володимирівна - к.т.н., доцент, доцент кафедри радіоелектронної автоматики, Дніпровського національного університету імені Олеся Гончара, Україна.

Астахов Дмитро Сергійович - старший викладач кафедри радіоелектронної автоматики, фізико-технічного факультету, Дніпровського національного університету імені Олеся Гончара, Україна.

Ogorenko Victoria - Doctor of medical sciences, associate professor,Professor, department of psychiatry, narcology and medical psychology, State institution "Dneprovsk medical academy of the ministry of health of Ukraine".

Klymenko Svetlana - candidate of technical sciences, associate professor,associate professor, department of radioelectronics automation, faculty of physics and technology, Oles Honchar dnipro national university, Ukraine.

Astakhov Dmitry - senior lecturer, department of radioelectronics automation, faculty of physics and technology, Oles Honchar dnipro national university, Ukraine. 